\title{
ANISOTROPIC ANGULAR BROADENING IN THE SOLAR WIND
}

\author{
A. K.R. ANANTHARAMAIAH \\ Raman Research Institute, Bangalore 560 080, INDIA \\ B. PRADEEP GOTHOSKAR \\ National Center for Radio Astrophysics, Pune 411 007, INDIA \\ and \\ C. T.J. CORNWELL \\ National Radio Astronomy Observatory, Socorro, NM 87801, USA
}

\begin{abstract}
.
We present Very Large Array observations at wavelengths of $2,3.5,6$, and $20 \mathrm{~cm}$, of angular broadening of radio sources due to the solar wind in the region 2-16 solar radii. Angular broadening is anisotropic with axial ratios in the range 2-16. Larger axial ratios are observed preferentially at smaller solar distances. Assuming that anisotropy is due to scattering blobs elongated along magnetic field lines, the distribution of position angles of the elliptically broadened images indicates that the field lines are non-radial even at the largest heliocentric distances observed here. At $5 R_{\odot}$, the major axis scattering angle is $\sim 0.7^{\prime \prime}$ at $\lambda=6 \mathrm{~cm}$ and it varies with heliocentric distance as $R^{-1.6}$. The level of turbulence, characterized by the wave structure function at a scale of $10 \mathrm{~km}$ along the major axis, normalized to $\lambda=20 \mathrm{~cm}$, has a value $20 \pm 7$ at $5 R_{\odot}$ and varies with heliocentric distance as $R^{-3}$. Comprison with earlier results suggest that the level of turbulence is higher during solar maximum. Assuming a power-law spectrum of electron density fluctuations, the fitted spectral exponents have values in the range 2.8-3.4 for scales sizes between $2-35 \mathrm{~km}$. The data suggests temporal fluctuations (of up to 10\%) in the spectral exponent on a time scale of a few tens of minutes. The observed structure functions at different solar distances do not show any evidence for an inner scale; the upper limits are $1 \mathrm{~km}$ at $2 R_{\odot}$ and $4 \mathrm{~km}$ at $13 R_{\odot}$. These upper limits are in conflict with earlier determinations and may suggest a reduced inner scale during solar maximum.
\end{abstract}

\section{Introduction}

Radio interferometric measurements of angular broadening is useful in determining several properties of the scattering medium. The Very Large Array (VLA), operated by NRAO, USA, has an excellent instantaneous UV coverage with baselines up to $35 \mathrm{~km}$ and multiple operating wavelengths $(\lambda=1.3$, $2,3.5,6,20,90 \mathrm{~cm})$, and is therefore well suited for studying the scattering phenomena in the solar wind, especially at small solar elongations $(2-20$ $\left.R_{\odot}\right)$. We have observed three radio sources using the VLA when the elongation of these sources varied between $0.5^{\circ}$ to $4^{\circ}$. At each of the observed positions these data provide information on the degree of anisotropy, orientation of the major axis and level of turbulence, which are, in turn, related to the orientation of the magnetic field and the magnitude of the density fluctuations in the solar wind. 
Observations were made using the A-configuration of the VLA during the period 2-6 Nov 1988. Three sources, 1430-155, 1437-153, and 1443162 , which are normally unresolved over the range of VLA baselines, were observed each day at two or more wavelengths chosen depending on the elongation. Due to many problems that are encountered while observing close to the Sun, the smallest elongation where useful data could be obtained at our longest observing wavelength $(\lambda=20 \mathrm{~cm})$ was $\sim 1.3^{\circ}$ and at the shortest wavelength $(\lambda=2 \mathrm{~cm})$ it was $\sim 0.5^{\circ}$. Each source was observed for about 10 minutes in the standard VLA continuum mode with a bandwidth of 50 $\mathrm{MHz}$ and an integration time of 10 seconds per visibility.

Images of the scatter broadened sources were obtained from the measured visibility data using standard radio synthesis methods. The scatterbroadened images are found to be highly anisotropic at small elongations in agreement with earlier findings (Hewish 1958, Armstrong et al 1990). We find that the axial ratios are in the range 2-16 depending on the elongation and position angle. The orientation of the major axis deviates significantly from being normal to the radial from the Sun's center. At some positions, the deviation is in the range $50-75^{\circ}$.

\section{Visibility Analysis}

The spatial coherence of the electric field described by the mutual coherence function is simply the normalized visibility given by $\Gamma(\mathbf{s})=\frac{V(\mathbf{s})}{V(0)}$ where $\mathbf{s}$ represents the baseline vector. The mutual coherence function lends itself to straightforward interpretation in terms of the scattering properties of the medium and also the spatial spectrum of electron density fluctuations in the medium. The relevant theory is discussed by Coles and Harmon (1989) and Armstrong et al (1990). Briefly, the mutual coherence is related to the wave structure function $D(\mathbf{s})$ by $\Gamma(\mathbf{s})=\exp \left[-\frac{D(\mathbf{s})}{2}\right]$. If the scattering medium is characterized by an electron density fluctuation spectrum of power-law form $P_{N_{e}}(q) \propto q^{-\beta}$ for $q_{\text {out }}<q<q_{\text {in }}$, where $q$ is the wavenumber, then the wave structure function also has a power law form given by $D(s) \propto s^{\beta-2}$ for $2<\beta<4$. Furthermore, at the inner scale $s_{i n}=2 \pi / q_{i n}$ the structure function breaks to the form $D(s) \propto s^{2}$. A plot of $\log D(s)$ versus $\log (s)$ can therefore be used to obtain the value of $\beta$ and also the inner scale if it falls within the range of baselines covered by the observations. Note that useful measurements of the structure function can be obtained only over the range of baselines covered by the observations and only when the scatter broadened source is partially resolved by the interferometric array.

Plots of $\log D(s)$ versus $\log (s)$, for all the observations, showed that (a) for a given position $\beta$ is independent of orientation, (b) there may be temporal variation in the value of $\beta$ on a time scale of few tens of minutes and (c) in all 
the cases there is no clear indication of an inner scale at which we expect the slope of $\log D(s)$ vs. $\log (s)$ to change from $\beta-2$ to 2 . We estimated upper limits to the inner scale as the shortest baseline down to which $\log D(s)$ shows a linear dependence on $\log (s)$.

\section{Discussion}

The observations presented here sample the scattering properties of the solar wind in the region $2-16 R_{\odot}$ and a range of heliographic latitudes. The parameters obtained are the magnitude of the scattering angle, the level of the structure function, the anisotropy of the scattering blobs, the orientation of the magnetic field lines, the spectral index $\beta$ of the electron density fluctuation spectrum and upper limits on the inner scale of the density flucuations. These quantities are summarized in Table 1 and briefly discussed below.

Table 1. Parameters as a function of solar distance ${ }^{\dagger}$

\begin{tabular}{|c|c|c|c|c|c|c|c|c|c|}
\hline $\begin{array}{c}R / R_{\odot} \\
(1)\end{array}$ & $\begin{array}{l}\text { Lat } \\
\left({ }^{\circ}\right) \\
(2)\end{array}$ & $\begin{array}{c}\phi_{\text {rad }} \\
\left({ }^{\circ}\right) \\
(3)\end{array}$ & $\begin{array}{c}\theta_{m a j}^{6 c m} \\
\left({ }^{\prime \prime}\right) \\
(4)\end{array}$ & (5) & $\begin{array}{c}D_{m a j}^{20} \\
(6)\end{array}$ & $\begin{array}{c}D_{m i n}^{20} \\
(7)\end{array}$ & $\begin{array}{c}<\beta> \\
(8)\end{array}$ & $\begin{array}{c}<P A> \\
\left(^{\circ}\right) \\
(9)\end{array}$ & $\begin{array}{c}s_{i n} \\
(\mathrm{~km}) \\
(10)\end{array}$ \\
\hline 1.72 & -15.3 & 128 & 9.50 & 3.8 & - & 560 & 2.78 & 46 & $<1.5$ \\
\hline 2.16 & 7.5 & 106 & 3.55 & 4.2 & 338 & 85 & 3.10 & 14 & $<1$ \\
\hline 2.64 & -28.3 & 142 & 0.44 & 4.5 & 19 & 6 & 3.00 & 83 & $<4$ \\
\hline 3.56 & -68.9 & 182 & 2.73 & 16.2 & 122 & 17 & 2.99 & 175 & $<1.5$ \\
\hline 4.76 & -80.1 & 193 & 0.60 & 3.8 & 21 & 5.5 & 2.97 & 156 & $<2$ \\
\hline 5.24 & -05.5 & 119 & 0.64 & 4.7 & 23 & 4 & 3.44 & 36 & $<5$ \\
\hline 5.84 & 10.0 & 291 & 1.43 & 10.6 & 64 & 5.0 & 3.25 & 22 & $<2$ \\
\hline 6.12 & 6.9 & 106 & 0.30 & 3.1 & 7.2 & 2.0 & 3.06 & 15 & $<4$ \\
\hline 8.40 & -31.3 & 250 & 0.31 & 6.6 & 5.0 & 0.8 & 3.14 & 178 & $<4$ \\
\hline 9.16 & -01.8 & 115 & 0.21 & 2.0 & 4.8 & 2.4 & 2.89 & 22 & $<2$ \\
\hline 9.84 & -07.4 & 289 & 0.18 & 3.8 & 3.4 & 1.2 & 2.90 & 23 & $<3.5$ \\
\hline 12.28 & -14.3 & 267 & 0.12 & 3.5 & 0.9 & 0.3 & 3.16 & 4 & $<6$ \\
\hline 13.16 & 0.1 & 113 & 0.08 & 2.9 & 0.8 & 0.3 & 3.11 & 27 & $<4$ \\
\hline 16.24 & -07.7 & 274 & 0.07 & 3.9 & 0.2 & 0.03 & 3.22 & 11 & $<8$ \\
\hline
\end{tabular}

$\dagger$ Errors are not quoted due to lack of space.

Please refer to Anantharamaiah et al (1994) for details 
Following Armstrong et al (1990), we characterize the level of turbulence by the magnitude of the structure function at a fixed scale of $10 \mathrm{~km}$ normalized to $\lambda=20 \mathrm{~cm}$. This quantity computed along the major and minor axis (denoted by $D_{\text {major }}$ and $D_{\text {minor }}$ ) of the scattered image are given in columns 6 and 7 of Table 1 . The turbulence level shows a power law behaviour; the fitted slopes are $-2.7 \pm 0.2$ for $D_{\text {major }}$ and $-3.0 \pm 0.2$ for $D_{\text {minor }}$. For a spherically symmetric solar wind having density variance proportional to the square of the mean density, the expected slope is -3.0 .

The angular sizes of the major and minor axes also show a power law dependance on solar distance $R$ with a slope of $-1.6 \pm 0.1 ; \theta_{m a j} \propto R^{-1.6}$ and is consistent with earlier findings (Lotova et al 1989). Angular broadening does not seem to depend on heliographic latitude. Presence of a transonic region in the solar wind between $10-20 R_{\odot}$ with enhanced scattering properties has been reported by Lotova et al (1989). The 3 data points that we have obtained in the transonic region do not show any excess scattering. Further observations are required to confirm the existence of such a region.

In the elongation range observed here $\left(2-16 R_{\odot}\right)$, all the scatter-broadened images show anisotropy with the axial ratio ranging from 2 to 16 . The magnitude of anisotropy does not show any strong depedance on solar distance, although larger axial ratios tend to occur only at smaller elongations. There could be a dependance of the axial ratio with heliographic latitude; images in the equatorial region seem to show less anisotropy. Anisotropy in the scattered image implies anisotropy of the scattering blobs which is perhaps caused due to the blobs being 'stretched' along magnetic field lines. The present data indicate that anisotropy is independent of scale size sampled by these osbervations (i.e. $1-35 \mathrm{~km}$ ).

The position angle of the minor axis of the scatter-broadened image indicates the direction along which the scattering blobs are stretched and therefore the local orientation of the magnetic field lines. The present data shows that the field lines deviate significantly from the radial direction in many of the positions observed. This may imply that, during solar maximum, the magnetic energy density could be comparable to the kinetic energy density even beyond $10 R_{\odot}$.

The derived values of the spectral index $\beta$ are in the range 2.8 to 3.4 with a mean of 3.1 (Table 1) which is significantly small compared to the Kolomogorov value of 3.67 . No dependance of $\beta$ is seen either with radial distance from the Sun (Fig 10d) or heliographic latitude. This value of the exponent applies to scale sizes in the range $2-35 \mathrm{~km}$ and is consistent with the values obtained by Coles and Harmon (1989) and Manoharan et al (1994). The upper limits to the inner scale (Column 11 of Table 1) at solar distances $8-16 R_{\odot}$ are more than a factor of two smaller than the inner scale values estimated by Coles and Harmon (1989) using spectral broadening 
data taken in 1984. If this difference is real, then we could speculate that the inner scales are smaller during a solar maximum.

Full details of these observations and additional discussions are published in Anantharamaiah et al (1994).

Acknowlledgement: The Very Large Array is a part of NRAO which is operated by Associated Universities Inc. under a co-operative agreement with the NSF.

\section{References}

Anantharamaiah K.R., Gothoskar, P. and Cornwell, T.J. 1994, J. Astrophys. Astr. 15, 387

Armstrong, J.W., Coles, W.A., Kojima, M. and Rickett, B.J. 1990, Astrophys. J., 358, 692

Coles, W.A. and Harmon, J.K. 1989, Astrophys. J., 337, 102

Goodman, J. and Narayan, R. 1989, Mon. Not. R. astr. Soc. 238995

Hewish, A. 1958, Mon. Not. Roy. astr. Soc., 118, 534

Lotova, N.A., Medvedeva, O.P., Kazimirskii, P.B., Sololeva, N.S. and Naugol'naya, M.N. 1989, Sov. Astron. 33, 333

Manoharan, P.K., Kojima, M. and Misawa, H. 1994, J. Geophys. Res. (in press)

Narayan, R., Cornwell, T.J., Goodman, J. and Anantharamaiah, K.R. 1990 in Radio Astronomical Seeing, ed. J.E. Baldwin and W. Shouguan, International Academic Press (Beijing) 